\title{
On Boyd-Wong-Type Fixed Point Results
}

\author{
Ivan D. Arandelović \\ Paper dedicated to memory of Vera Nikolić-Stanojević, \\ Pofessor Emeritus at the State University of Novi Pazar
}

\begin{abstract}
This talk (paper) gives a survey of recent results in the theory of Boyd-Wong-type contractions and its aim is to present a simple and unified treatment to this theory. In final part of paper, we present one recent fixed point result for Boyd-Wong-type contractions defined on symmetric spaces.
\end{abstract}

Keywords: fixed point, Picard iterates, symmetric space

\section{Introduction}

Let $X$ be a nonempty set and $f: X \rightarrow X$ arbitrary mapping. $x \in X$ is a fixed point for $f$ if $x=f(x)$. If $x_{0} \in X$, we say that a sequence $\left(x_{n}\right)$ defined by $x_{n}=f^{n}\left(x_{0}\right)$ is a sequence of Picard iterates of $f$ at point $x_{0}$ or that $\left(x_{n}\right)$ is the orbit of $f$ at point $x_{0}$.

The contraction mapping principle, formulated and proved in $\mathrm{Ph}$. D. dissertation of S. Banach in 1920 which was published in 1922 is one of the most important theorems in classical functional analysis because it give:

1. the existence and uniqueness of fixed point,

2. method to obtain approximative fixed points,

3. error estimates for approximative fixed point obtained by 2 .

There are many generalizations and partial generalizations (which consider only statements 1. and 2.) of the Banach principle. One of the most important is fixed point theorem of Boyd and Wong [3] (Nonlinear contraction principle). In this talk (paper) we applied Rus's [8] lemma to nonlinear contractions and so obtained one extension of Boyd - Wong's result.

There have been a number of generalizations of metric space. One of them is the notion of symmetric spaces introduced by M. Fréchet, K. Menger, E. W. Chittenden and W. A. Wilson. M. Cicchese introduced the notion of a contraction mapping in symmetric spaces and

\footnotetext{
Manuscript received May 30, 2014. ; accepted October 21, 2014.

Ivan D. Aranđelović is with the University of Belgrade - Faculty of Mechanical Engineering Kraljice Marije 16, 11000 Beograd, Serbia.
} 
proved the first fixed point theorem for this class of spaces. Further fixed point results for this class of spaces was obtained by J. Jachymski, J. Matkowski and T. Swiatkowski; T. L. Hicks and B. E. Rhoades; M. Aamri and D. El Moutawakil; M. Aamri, A. Bassou and D. El Moutawakil; J. Zhu, Y. J. Cho and S. M. Kang; D. Miheţ; M. Imdad, J. Ali and L. Khan; A. Aliouche,... For additional informations and references see [1].

In final part of paper, we present one recent fixed point result for Boyd-Wong-type contractions defined on symmetric spaces.

\section{Comparison functions}

Let $(X, d)$ be a metric space, $\varphi:[0, \infty) \longrightarrow[0, \infty)$ and $\varphi(0)=0$. Define:

1) $\varphi \in \Phi_{0}$ if and only if $\varphi(r)<r$ for each $r>0$;

2) $\varphi \in \Phi_{1}$ if and only if $\lim _{n \rightarrow \infty} \varphi^{n}(r)=0$ for each $r>0$;

3) $\varphi \in \Phi_{2}$ if and only if $\varphi$ is monotone nondecreasing;

4) $\varphi \in \Phi_{3}$ if and only if $\varphi$ is continuous from the right on $(0,+\infty)$;

5) $\varphi \in \Phi_{4}$ if and only if $\varlimsup_{t \rightarrow r+} \varphi(t)<r$ za svako $r>0$;

6) $\varphi \in \Phi_{5}$ if and only if $\varlimsup_{t \rightarrow \infty}(t-\varphi(t))=\infty$;

7) $\varphi \in \Phi_{6}$ if and only if $\sum_{n=1}^{\infty} \varphi^{n}(t)<\infty$ for each $r>0$;

8) $\varphi \in \Phi_{a}$ if and only if $\varphi \in \Phi_{0} \cap \Phi_{1}$;

9) $\varphi \in \Phi_{b}$ if and only if $\varphi \in \Phi_{1} \cap \Phi_{2}$;

11) $\varphi \in \Phi_{c}$ if and only if $\varphi \in \Phi_{1} \cap \Phi_{9}$

12) $\varphi \in \Phi_{d}$ if and only if $\varphi \in \Phi_{0} \cap \Phi_{2} \cap \Phi_{3} \cap \Phi_{5}$;

13) $\varphi \in \Phi_{e}$ if and only if $\varphi \in \Phi_{0} \cap \Phi_{4}$;

where $\varphi^{n}=\varphi \circ \cdots \circ \varphi$.

\section{Nonlinear contractions}

Let $(X, d)$ be a metric space, $x \in X$ and $r>0$. By $\overline{B(x, r)}$ we denote

$$
\{z \in X: d(z, x) \leqslant r\} .
$$

Now we need the following lemma.

Lemma 3.1 (I. Rus [8]) Let $(X, d)$ be a complete metric space and $f: X \rightarrow X$. Suppose that for each $\varepsilon>0$ there exists $\delta(\varepsilon)$ such that, for each $x \in X$

$$
d(x, f(x))<\delta(\varepsilon) \text { implies } f(\overline{B(x, r)}) \subseteq \overline{B(x, r)} .
$$

The for each $x_{0} \in X$ such that $\lim d\left(f^{n}\left(x_{0}\right), f^{n+1}\left(x_{0}\right)\right)=0,\left(f^{n}\left(x_{0}\right)\right)$ is a Cauchy sequences and its limit is fixed point of $f$.

In this section our main result is the following theorem. 
Theorem 3.1 Let $(X, d)$ be a complete metric space, $\varphi \in \Phi_{a}$ and $f: X \rightarrow X$. If

$$
d(f(x), f(y)) \leqslant \varphi(d(x, y)) \text { for each } x, y \in X ;
$$

then $f$ has unique fixed point $z \in X$ and all sequences of Picard iterates defined by $f$ converges to $z$.

Proof: Let $x, y \in X$. Then from

$$
d\left(f^{n}(x), f^{n}(y)\right) \leqslant d\left(f^{n}(x), f^{n+1}(x)\right)+d\left(f^{n+1}(x), f^{n+1}(y)\right)+d\left(f^{n}(y), f^{n+1}(y)\right),
$$

we get that

$$
d\left(f^{n}(x), f^{n}(y)\right) \leqslant \varphi^{n}\left(d(x, f(x))+\varphi^{n+1}(d(x, y))+\varphi^{n}(d(y, f(y)) .\right.
$$

So we obtain that

$$
\lim d\left(f^{n}(x), f^{n}(y)\right)=0,
$$

for any $x, y \in X$, which implies that all sequences of Picard iterates defined by $f$ are equiconvergent. Hence, for arbitrary $x \in X$ and $y=f(x)$ we obtain that sequences $\left(f^{n}\left(x_{0}\right)\right)$ and $\left(f^{n+1}\left(x_{0}\right)\right)$ are equiconvergent, which implies that $\lim d\left(f^{n}(x), f^{n+1}(x)\right)=0$.

Let $x \in X \varepsilon>0$ be arbitrary. Define $\delta(\varepsilon)=\varepsilon-\varphi(\varepsilon)$. For any $y \in \overline{B(x, r)}$ we get that

$$
d(f(y), x) \leqslant d(f(y), f(x))+d(x, f(x)) \leqslant \varphi(\varepsilon)+d(x, f(x)) .
$$

Hence

$$
d(x, f(x))<\delta(\varepsilon) \text { implies } f(\overline{B(x, r)}) \subseteq \overline{B(x, r)} .
$$

By Lemma 3.1 we obtained that sequences of Picard iterates defined by $f$ converges to some fixed point of $f$. Such fixed point must be unique because sequences of Picard iterates are equiconvergent.

Theorem 3.1 generalize earlier results obtained by F. Browder [4] (it has assumption $\varphi \in \Phi_{d}$ ), R. M. Bianchini and M. Grandolfi [2] $\varphi \in \Phi_{c}$ ), D. W. Boyd, and J. S. W. Wong [3] (it has assumption $\varphi \in \Phi_{e}$ ) and A. Zitarosa [9] (it has assumption $\varphi \in \Phi_{b}$ ).

\section{Convergence of Picard iterations}

Hicks and Rhoades [5] proved that many known generalizations of the Banach principle were included in the following result (see also [7]).

Theorem 4.1 (Hicks and Rhoades [5]) Let $(X, d)$ be a metric space and $f: X \longrightarrow X$. If there exists real number $\alpha \in(0,1)$ such that

$$
d\left(f(x), f^{2}(x)\right) \leqslant \alpha d(x, f(x))
$$

for every $x \in X$, then each sequence of Picard iterates defined by $f$ is a Cauchy sequence. Furthermore, if $X$ is complete and if a mapping $G(x)=d(x, f(x))$ is lower semi-continuous at a limit point of $\left\{f^{n}(x)\right\}$, say $x^{*}$, then $x^{*}$ is a fixed point of $f$. 
Proof of this Theorem can be easily selected from the proof of Banach's theorem.

In following Propositions we shall prove that in the statement of Theorem 1 condition 4.1 can not be replaced with

$$
d\left(f(x), f^{2}(x)\right) \leqslant \varphi(d(x, f(x))
$$

where comparison function $\varphi \in \Phi_{0} \cap \Phi_{1} \cap \Phi_{2} \cap \Phi_{3} \cap \Phi_{4} \cap \Phi_{5}$.

Proposition 4.1 There exists complete metric space $(X, d), f: X \longrightarrow X$ and comparison function $\varphi \in \Phi_{0} \cap \Phi_{1} \cap \Phi_{2} \cap \Phi_{3} \cap \Phi_{4} \cap \Phi_{5}$, which satisfies condition (4.2), such that all sequences of Picard iterates defined by $f$ are divergent.

Proof: Let $X=[0, \infty)$ with usually metrics, $f(x)=\ln (1+\exp (x))$ and

$$
\varphi(t)=\ln (2-\exp (-t)) \text {. }
$$

Then: $g(x)=d(x, f(x))=\ln (1+\exp (-x))$,

$h(x)=d\left(f(x), f^{2}(x)\right)=\ln \left(1+(1+\exp (x))^{-1}\right), \varphi(t)=h\left(g^{-1}(t)\right)$

and $d\left(f(x), f^{2}(x)\right)=h(x)=h\left(g^{-1}(g(x))\right)=\varphi(d(x, f(x))$. We can see that $f$ has no fixed point and its sequences of Picard iterates are divergent. $\diamond$

Proposition 4.2 There exists bounded complete metric space $(X, d), f: X \longrightarrow X$ and comparison function $\varphi \in \Phi_{0} \cap \Phi_{1} \cap \Phi_{2} \cap \Phi_{3} \cap \Phi_{4} \cap \Phi_{5}$, which satisfies condition (4.2), such that all sequences of Picard iterates defined by $f$ are divergent.

Proof: Let $\left(x_{n}\right)$ be arbitrary sequence, $X=\left\{x_{n}\right\}$ and

$d: X^{2} \rightarrow[0,+\infty)$ mapping defined by

$$
d\left(x_{n}, x_{n+k}\right)=\frac{k}{n+k},
$$

$n \in\{1,2,3, \ldots\}, k \in\{0,1,2, \ldots\}$. Now define $f: X \rightarrow X$ by $f\left(x_{n}\right)=x_{n+1}$, and $\varphi:[0, \infty) \rightarrow$ $[0, \infty)$ by $\varphi(t)=\frac{x}{x+1}$.

$(X, d)$ is complete bounded metric space, because each ball which radius is less then 1 contains only a finite number elements of $X$. Inequality (4.2) are also satisfied, but all sequences of Picard iterates defined by $f$ are divergent.

\section{Nonlinear contractions on symmetric spaces}

Let $X$ be a non-empty set and $d: X^{2} \rightarrow[0, \infty) .(X, d)$ is symmetric space (also called $E$ space, in terminology of M. Fréchet) if and only if it satisfies:

(W1) $d(x, y)=0$ if and only if $x=y$;

(W2) $d(x, y)=d(y, x)$ for any $x, y \in X$.

Symmetric spaces differ from more convenient metric spaces in the absence of triangle inequality. Nevertheless, many notions can be defined similar to those in metric spaces. For instance, in symmetric space $(X, d)$ limit point of a sequence $\left(x_{n}\right)$ is defined by

$$
\lim d\left(x_{n}, x\right)=0 \Leftrightarrow \lim x_{n}=x .
$$


Also, a sequence $\left\{x_{n}\right\} \subseteq X$ is said to be a Cauchy sequence, if for every given $\varepsilon>0$, there exists a positive integer $n_{0}$ such that $d\left(x_{m}, x_{n}\right)<\varepsilon$ for all $m, n \geqslant n_{0}$. A symmetric space $(X, d)$ is said to be complete if and only if each its Cauchy sequence converges to some $x \in X$.

Diameter of $A \subseteq X$ is defined by

$$
\operatorname{diam}(A)=\sup _{x, y \in A} d(x, y),
$$

whereas the open ball with center $x \in X$ and radius $r>0$ is defined by

$$
B(x, r)=\{y \in X: d(x, y)<r\} .
$$

In the literature there are many conditions which can be used as a partial replacement for triangle inequality. Now we present some of them.

Definition 5.1 Let $(X, d)$ be a symmetric space. We define the following properties:

(W3) $\lim d\left(x_{n}, x\right)=0 \wedge \lim d\left(x_{n}, y\right)=0 \Rightarrow x=y$;

$(\mathrm{JMS}) \lim d\left(x_{n}, y_{n}\right)=0 \wedge \lim d\left(y_{n}, z_{n}\right)=0 \Rightarrow \varlimsup \lim d\left(x_{n}, z_{n}\right) \neq \infty$.

Next statement gives the characterization of symmetric space which satisfies the property (JMS).

Proposition 5.1 (J. Jachymski, J. Matkowski and T. Swiatkowski [6]) Let (X, d) be a symmetric space. Then the following conditions are equivalent.

(i) $(X, d)$ satisfies property (JMS);

(ii) There exists $\delta, \eta>0$ such that for any $x, y, z \in X$,

$$
d(x, z)+d(z, y)<\delta \text { implies that } d(x, y)<\eta .
$$

(iii) There exists $r>0$ such that

$$
\sup \{\operatorname{diam}(B(x, r)): x \in X\}<\infty .
$$

Now, we present one recent fixed point result for Boyd-Wong-type contractions defined on symmetric spaces. This Theorem generalize earlier result obtained by J. Jachymski, J. Matkowski and T. Swiatkowski [6] for class of semi-metric spaces.

Theorem 5.1 Let $(X, d)$ be a complete symmetric space which satisfies the properties (W3) and (JMS), $f: X \rightarrow X$ and $\varphi \in \Phi_{b}$. If

$$
d(f(x), f(y)) \leqslant \varphi(d(x, y))
$$

for any $x, y \in X$, then $f$ has a unique fixed point $y \in X$ and for each $x \in X$ the sequence of Picard iterates defined by $f$ at $x$ converges to $y$.

The following problem is still open.

Problem 5.1 Does Theorem (5.1) hold if $\varphi \in \Phi_{b}$ is replaced with $\varphi \in \Phi_{a}$ ?

Acknowledgement. Author was partially supported by Ministry of Education and Science Grant, No. 174002, Serbia. 


\section{References}

[1] I.D. ARAND̄Elović, D.J. KEČKić, Symmetric spaces approach to some fixed point results, Nonlinear Analysis 75 (2012), 5157-5168.

[2] R. M. Bianchini, M. GRandolfi, Transformazioni di tipo contracttivo generalizzato in uno spazio metrico, Atti Accad. Naz. Lincei, VII. Ser. Rend. Cl. Sci. Fis. Mat. Natur. 45 (1968) 212-216.

[3] D. W. Boyd, J. S. W. Wong, On nonlinear contractions, Proc. Amer. Math. Soc. 20 (1969) 458-464.

[4] F. BROWDER, On the convergence of successive approximations for nonlinear functional equations, Indag. Math. 30 (1968) 27-35.

[5] T. L. Hicks, B. E. RHOADES, A Banach type fixed point theorem, Math. Japonica 24, (1979) 327-330.

[6] J. JACHYMSKI, J. MATKOWSKI AND T. ŚWIATKOWSKI, Nonlinear contractios on semimetric spaces, J. Appl. Anal. 1 (1995), 125-134.

[7] B. E. RhoAdes, Contractive definitions revisited, Contemporary Mathematics 21 (1983) 189-205.

[8] I. Rus, Generalized contractions, "Babes-Bolyai" University, Faculty of Mathematics, Reasrch Seminaries, Seminary on Fixed Point Theory, Preprint nr. 3 (1983), 1-1130.

[9] A. Zitaros A, na generalizzatione del teorema di Banach sulle contrazioni, Mathematicae 23 (1968) 417-424. 